\title{
Knocking down the expression of Aurora-A gene inhibits cell proliferation and induces G2/M phase arrest in human small cell lung cancer cells
}

\author{
YIMIN LU ${ }^{1,2}$, YUANHUA LIU ${ }^{3}$, JIANZHONG JIANG $^{4}$, ZHAOQING XI $^{1}$, NING ZHONG $^{5}$, \\ SHUNBIN SHI $^{6}$, JINZHI WANG ${ }^{7}$ and XIAODONG WEI ${ }^{8}$
}

${ }^{1}$ Department of Emergency, Jiangsu Province Hospital of TCM, Affiliated Hospital of Nanjing University of Chinese Medicine, Nanjing, Jiangsu 210029; ${ }^{2}$ Department of Respiratory Medicine, Kunshan First People's Hospital Affiliated to Jiangsu University, Kunshan, Jiangsu 215300; ${ }^{3}$ Jiangsu Cancer Hospital and Jiangsu Institute of Cancer Research, Nanjing, Jiangsu 210009; ${ }^{4}$ Department of Geriatrics, People's Hospital of Yixing City, Yixing, Jiangsu 214200; ${ }^{5}$ Department of Surgery, Kunshan First People's Hospital Affiliated to Jiangsu University, Kunshan, Jiangsu 215300; ${ }^{6}$ Department of Thoracic Surgery, Affiliated Wujiang Hospital of Nantong University, Suzhou, Jiangsu $215200 ;{ }^{7}$ Department of Cell Biology,

School of Medicine, Soochow University, Suzhou, Jiangsu $215123 ;{ }^{8}$ Department of Thoracic Surgery,

No. 101 Hospital of PLA, Wuxi, Jiangsu 214044, P.R. China

Received February 7, 2014; Accepted March 10, 2014

DOI: 10.3892/or.2014.3194

\begin{abstract}
A hallmark of small cell lung cancer (SCLC) is frequent relapse characterized by newfound resistance to formerly efficacious chemotherapies. The prognosis for SCLC patients is particularly unfavorable. Aurora kinase A (AURKA), a member of the serine/threonine kinase family, is overexpressed across many types of human tumors. Recent studies have identified AURKA as an important factor in tumorigenesis, but little is known regarding its specific roles in SCLC. The aim of the present study was to establish the roles of AURKA in the molecular pathogenesis of human SCLC. In the present study, we constructed a lentiviral vector to express siRNA against AURKA (LV-AURKA siRNA). As we expected, the viral construct effectively suppressed the expression of the AURKA gene and protein in H446 and H1688 cell lines. Additionally, RNA interference of AURKA inhibited the colony formation and subsequent growth of H446 and H1688 cell lines by increasing the incidence of cell cycle arrest in the G2/M phase. Furthermore, suppression of AURKA by LV-AURKA siRNA also increased apoptosis of SCLC cells. A potential mechanism for the increase of apoptosis is the downregulation of Bcl-2 and upregulation of Bax. AURKA
\end{abstract}

Correspondence to: Professor Xiaodong Wei, Department of Thoracic Surgery, No. 101 Hospital of PLA, Wuxi, Jiangsu 214044, P.R. China

E-mail: 120504141@qq.com

Dr Jinzhi Wang, Department of Cell Biology, School of Medicine, Soochow University, Suzhou, Jiangsu 215123, P.R. China

E-mail: 451588568@qq.com

Key words: lentivirus, RNA interference, Aurora-A, small cell lung cancer cells gene suppression may provide a novel, effective therapy for SCLC patients by inhibiting cell division and increasing the rate of apoptosis of SCLC cells.

\section{Introduction}

Lung cancer is the leading cause of cancer-related mortality worldwide. Small cell lung cancer (SCLC) accounts for $13 \%$ of lung cancer-related deaths and $12 \%$ of deaths from all other types of cancer $(1,2)$. SCLC is recognized for its aggressiveness, displaying rapid growth throughout and early, widespread metastasis (3). In addition, chemotherapy does not consistently prolong the survival of SCLC patients, especially following relapse, as demonstrated by several previous studies (4). Aside from the lack of efficacy of current treatments, the relatively few molecules identified for precise targeting is a key contributing factor to the low survival rate. Thus, understanding the molecular underpinnings of SCLC, and revealing effective treatment options for SCLC, are necessary to improve outcomes for SCLC patients.

Aurora kinase A (AURKA) is a centrosome-associated serine/threonine kinase. AURKA participates in several crucial mitotic events, including centrosome maturation and separation, formation of the bipolar spindle, chromosome alignment and segregation, and cytokinesis $(5,6)$. Performing an essential role as a facilitator in cell cycle progression, AURKA is an important oncogene. The AURKA is located in chromosome 20q13.2. This region is frequently amplified in a variety of cancers. Also, AURKA overexpression is identified in gastric, breast, ovarian, colorectal and esophageal tissues (7). Overexpression of AURKA has been shown to paralyze the $\mathrm{G} 2 / \mathrm{M}$ checkpoint and spindle assembly checkpoints, enabling rogue cells with defective spindles and damaged DNA to enter mitosis and anaphase. These defects 
likely contribute to genomic instability, and ultimately, carcinogenesis $(8,9)$. Centrosome amplification and aneuploidy, two additional molecular drivers of genomic instability and tumorigenesis, have also been associated with the overexpression of AURKA $(10,11)$.

In the last decade, RNA interference (RNAi) has served as a powerful tool for precisely inhibiting selective gene expression. The application of RNAi to medicine is a promising treatment option for various diseases, especially cancer $(12,13)$. The introduction of nucleic acids such as non-coding, synthetic small interfering RNAs (siRNAs) can modulate the expression of target genes in a sequence-dependent manner. This occurs through two mechanisms that siRNA incorporates into RNA-induced silencing complexes (RISCs), and thereafter actively degrades and represses the translation of corresponding messenger RNAs (mRNAs) $(14,15)$.

There is little knowledge regarding the function of AURKA in SCLC cells. In the present study, we knocked down AURKA gene in two SCLC cell lines, H446 and H1688, and the apoptosis of these cells increased through downregulation of Bcl-2 and upregulation of Bax, which provides new insights into the roles of AURKA in SCLC treatment.

\section{Materials and methods}

Cell culture. The human SCLC cell lines H446 and H1688 were maintained in Dulbecco's modified Eagle's medium (DMEM) (Gibco, Grand Island, NY, USA) supplemented with $10 \%$ fetal bovine serum (FBS), $100 \mathrm{U} / \mathrm{ml}$ penicillin and $0.1 \mathrm{mg} / \mathrm{ml}$ streptomycin under normoxic conditions $\left(95 \% \mathrm{O}_{2}\right.$ and $5 \% \mathrm{CO}_{2}$ ) at $37^{\circ} \mathrm{C}$.

Cell growth inhibition assay. The effects of AURKA shRNA infection on the proliferation of H446 and H1688 cells were analyzed by methylthiazolyldiphenyl-tetrazolium bromide (MTT) assay. The SCLC cells were seeded into a 96-well plate at a concentration of $5 \times 10^{3}$ cells/well for several different time courses. A total of $20 \mu \mathrm{l}$ of MTT (Sigma, St. Louis, MO, USA) stock solution ( $5 \mathrm{mg} / \mathrm{ml})$ was added to each well and the cultures were incubated at $37^{\circ} \mathrm{C}$ for an additional $4 \mathrm{~h}$. This period of time was sufficient for formazan crystals to precipitate. These were then dissolved by adding $150 \mu$ l of dimethyl sulfoxide (DMSO) to each well. The optical density was read by an enzyme-linked immunosorbent assay (490 nm).

Recombination lentivirus generation. The sequence for human AURKA specific small interfering RNA (siRNA) sequence is 5'-GAAAGCTCCACATCAATAA-3'; this was designed with the sequence generator accessible on Invitrogen's website, using the AURKA sequence as a reference (GeneBank code: NM_003600). The non-silencing (NS) sequence (5'-TTCTCC GAACGTGTCACGT-3') was used as a random, scrambled control. The short hairpin RNA (shRNA) cassette against AURKA was: 5'-CCGGCAGAAAGCTCCACATCAATAAT TCAAGAGATTATTGATGTGGAGCTTTCTGTTTTTG-3', with two cohesive ends for ligation into the pGCSIL-GFP vector. Pairs of complementary oligonucleotides of these sequences were ligated into the pGCSIL-GFP vector. The constructed lentiviral plasmid was hereafter denoted as pGCSIL-GFP-sh AURKA. By co-transfection of pGCSIL-
GFP-shAURKA into pHelper 1.0 and pHelper 2.0 plasmids, AURKA and lentivirus were re-generated in 293T cells. The final lentiviral construct was referred to as LV-AURKA siRNA or AURKA-siRNA. We generated lentiviruses that express non-silencing, scrambled shRNA as a control, which we refer to as scr-siRNA. The SCLC cells were transfected with the AURKA siRNA and scramble siRNA, respectively.

Lentivirus infection. Cells were incubated with lentivirus in a small volume of serum-free DMEM at $37^{\circ} \mathrm{C}$ for $4 \mathrm{~h}$. DMEM containing $10 \%$ FBS was then added and cells were placed in the incubator for an additional period of time, as indicated, for the remainder of the experiment. Green fluorescent protein (GFP) showed that the infection efficiency in SCLC cells was $\sim 90 \%$ at a multiplicity of infection (MOI) of 30, no viral cellular toxicity was noted at this concentration of lentivirus. Thus, the following experiments were performed using this concentration to achieve MOI of 30, except where indicated otherwise.

Real-time PCR analysis. The mRNA of AURKA in SCLC cells was analyzed by real-time PCR. RNA was initially extracted from lentivirus-infected SCLC cells 5 days postinfection using TRIzol (Invitrogen). Reverse transcription was performed using a Promega M-MLV cDNA synthesis kit according to the manufacturer's instructions. Real-time qPCR analysis was performed using the SYBR-Green Master Mix kit and the DNA Engine Opticon ${ }^{\mathrm{TM}}$ System (MJ Research, Waltham, MA, USA). The expression of glyceraldehyde 3-phosphate dehydrogenase (GAPDH) was used as an internal control. The forward and reverse primers of AURKA were: 5'-GCC CTG TCT TAC TGT CAT TCG-3' and 5'-AGG TCT CTT GGT ATG TGT TTG C-3', respectively. The forward and reverse primers of GAPDH were: 5'-TGA CTT CAA CAG CGA CAC CCA-3' and 5'-CAC CCT GTT GCT GTA GCC AAA-3', respectively. The relative gene expression levels were calculated using the $2^{-\Delta \Delta \mathrm{CT}}$ algorithm.

Colony-forming assay. A soft agar colony formation assay was performed to assess the anchorage-independent growth ability of SCLC cells as a characteristic of in vitro tumorigenicity. The infected SCLC cells were counted and inoculated in 6 -well plates at a density of 500 cells/well. Following incubation for 14 days, the SCLC cells were immobilized by $4 \%$ paraformaldehyde and stained using Giemsa dye for $20 \mathrm{~min}$. Then, the cells were rinsed with distilled water and colonies were counted and photographed under a microscope.

Flow cytometry analysis. The SCLC cells were briefly seeded and infected with virus in stock medium. The stock medium was then removed and replaced with a serum-free medium for $48 \mathrm{~h}$ incubation. After the time period, adherent cells were harvested by trypsinization and suspended in $0.5 \mathrm{ml}$ of $70 \%$ alcohol. They were briefly subjected to $4^{\circ} \mathrm{C}$ conditions for $30 \mathrm{~min}$. The cells were then incubated with $50 \mu \mathrm{g} / \mathrm{ml}$ of propidium iodide (PI; Sigma) for $1 \mathrm{~h}$ in the dark. A total of $1.0 \times 10^{6}$ SCLC cells were washed twice with ice-cold PBS and incubated for $15 \mathrm{~min}$ in $100 \mu \mathrm{l}$ staining buffer that included $5 \mu \mathrm{l}$ APC labeled Annexin V. Fluorescence-activated cell sorting (FACS) analysis for Annexin V staining was 


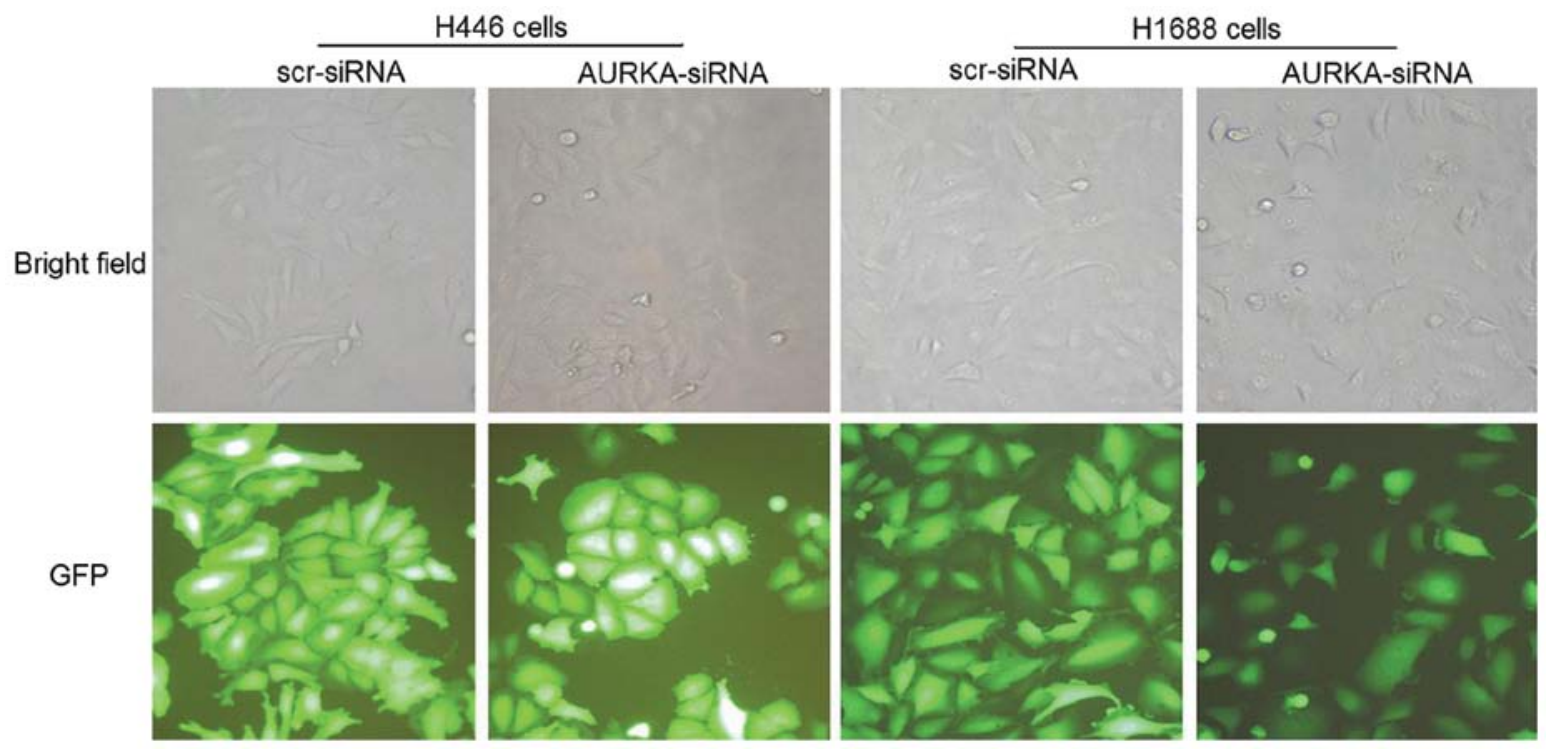

Figure 1. Qualitative analysis of lentivirus infection efficiency in SCLC cells. Green fluorescence indicates the successful delivery of siRNAs into H446 and H1688 cells, respectively. At MOI 30, 90\% cells were infected. SCLC, small cell lung cancer; siRNAs, small interfering RNAs; MOI, multiplicity of infection.

performed by flow cytometer. The DNA content of stained nuclei was analyzed by a flow cytometer using MultiCycle DNA cell cycle analyzed software to determine the percentage of cells for each phase of the cell cycle. Each experiment was performed in triplicate.

Western blot analysis. The SCLC cell pellets were lysed with protein extraction solution and incubated at $-20^{\circ} \mathrm{C}$ for $20 \mathrm{~min}$. The cell lysates were then centrifuged at 12,000 x $\mathrm{g}$ for $5 \mathrm{~min}$ and total proteins were collected.

After protein quantization using a Coomassie brilliant blue assay, $50 \mu \mathrm{g}$ of protein was separated by $10 \%$ SDS-PAGE and transferred to a polyvinylidene fluoride (PVDF) membrane. The membrane was blocked with 5\% skim milk and proteins were detected with primary antibodies against Aurora-A, Bcl-2, Bax, CDK1 and GAPDH. The primary antibody was detected with horseradish peroxidase-conjugated anti-mouse for $1 \mathrm{~h}$ at $25^{\circ} \mathrm{C}$. The horseradish peroxidase-conjugated secondary antibody was visualized with enhanced chemiluminescence (ECL; Millipore, Bedford, MA, USA). Protein band intensities were determined using the video imaging CMIASWIN system (Bio-Rad, Hercules, CA, USA). GAPDH was once more used as an internal positive control.

Statistical analysis. Data are represented as a means \pm SD of results from the three independent experiments with similar patterns. Statistical significance of difference between AURKA-siRNA and scr-siRNA groups was determined by Student's t-test and one-way ANOVA using GraphPad Prism 5.0 software. For all experiments, $\mathrm{p}<0.05$ was considered to indicate a statistically significant difference.

\section{Results}

Construction of lentivirus vector mediating RNAi targeting of AURKA (LV-AURKA siRNA) and its effects on AURKA expression. To elucidate the role of AURKA in SCLC, we first constructed lentivirus vectors to deliver either AURKA-specific siRNA or non-specific scramble-siRNA. H446 and H1688 SCLC cell lines were randomly infected with one of the vector types. To investigate the lentiviral infection efficiency, an immunofluorescence assay was conducted, which showed that $>90 \%$ of the cells exhibited the green fluorescence indicative of infection (Fig. 1). To confirm whether the AURKA-siRNA had silenced the expression of AURKA and dampened AURKA protein levels, real-time qPCR and western blot analyses were performed on the lentivirus-infected cells. The results indicated that the quantity of AURKA mRNA (Fig. 2A) and protein (Fig. 2B) were significantly reduced by the infection of AURKA-siRNA in both H446 and H1688 cells compared to scr-siRNA treatment groups. These results suggest that the AURKA-siRNA effectively suppressed the AURKA expression in SCLC cells.

Effects of AURKA-siRNA on cell proliferation, colony formation. An MTT assay was performed to study the effect of AURKA-siRNA on H446 and H1688 cell growth. As shown in Fig. 3, both H446 (Fig. 3A) and H1688 cells (Fig. 3B) showed significant $(\mathrm{p}<0.01)$ reduction in cell viability. These results indicate that the proliferation rate of cells is significantly dampened when the AURKA gene was silenced. AURKA is a facilitator of cell division, and the frequency of cell division mirrors the magnitude of AURKA protein presence in the cell in a parallel fashion.

Heightened colony formation is an essential morphologic feature of the SCLC cells. The result of a colony formation assay showed that in $\mathrm{H} 446$ cells the number of colonies of the AURKA-siRNA group $(14 \pm 1)$ was significantly less than that of the scr-siRNA group $(44 \pm 4)(\mathrm{p}<0.01)$ (Fig. 4A). In H1688 cells, the number of colonies of the AURKA-siRNA group was $1 \pm 1$, markedly lower than that in the scr-siRNA group $(41 \pm 2)$ $(\mathrm{p}<0.01)$ (Fig. 4B). These results demonstrated that a reduction in AURKA expression resulted in a decreased ability of the H446 and H1688 cell types to form colonies. 
A

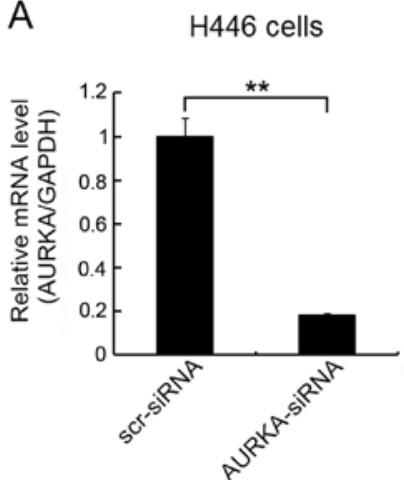

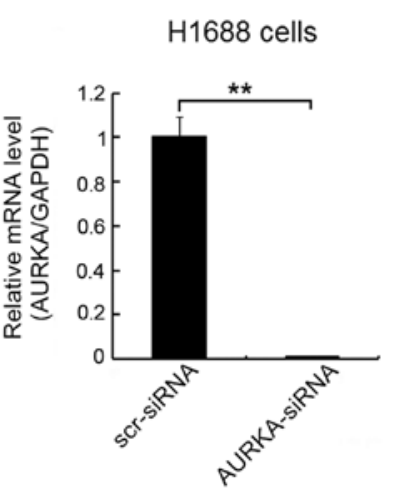

B

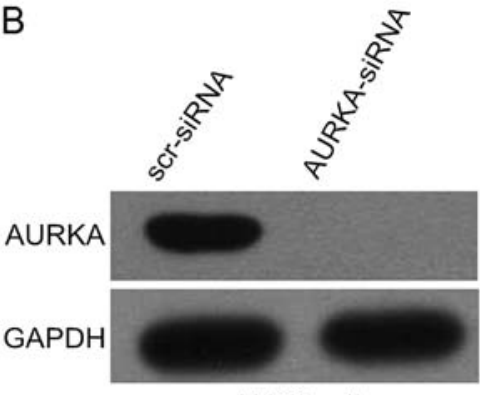

H466 cells

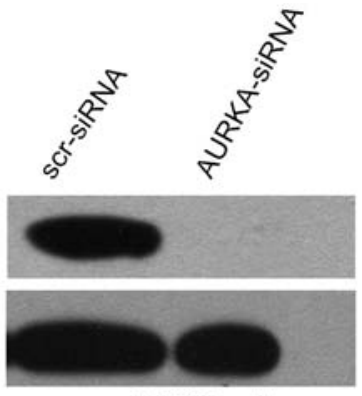

H1688 cells

Figure 2. The AURKA gene knocked down in SCLC cells. (A) The mRNA of AURKA gene was measured by qPCR after AURKA-siRNA and scr-siRNA treatment in H446 and H1688 cells, respectively. Protein levels of AURKA under these two separate treatments in H446 and H1688 cells were analyzed by western blotting. (B) The AURKA protein could not be detected in H446 and H1688 cells after AURKA-siRNA treatment. ${ }^{* *}$ p $<0.01$, compared with control treatment. AURKA, Aurora kinase A; SCLC, small cell lung cancer; siRNA, small interfering RNA.

A

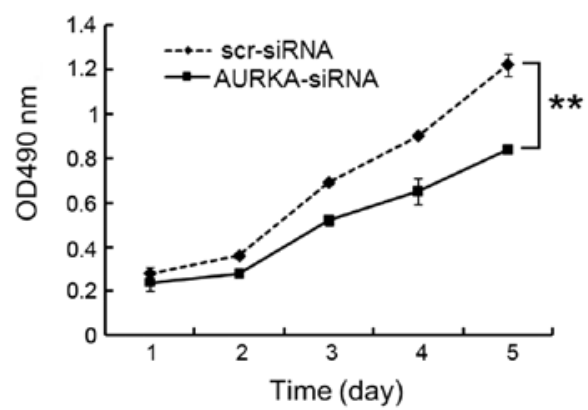

B H1688 cells

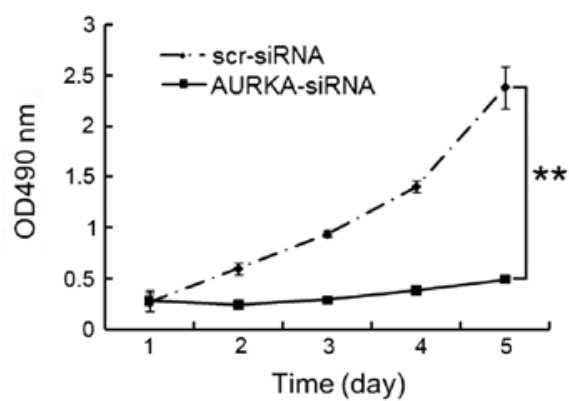

Figure 3. Effect of AURKA silencing on the proliferation of SCLC cells. The proliferation rates were measured by MTT assay in AURKA-silenced and non-silenced H446 (A) and H1688 (B) cells. LV-AURKA siRNA suppressed the growth curves of SCLC cells as compared with scr-RNA determined by MTT assay.** $\mathrm{p}<0.01$, compared with control treatment. AURKA, Aurora kinase A; SCLC, small cell lung cancer; siRNA, small interfering RNA.

A

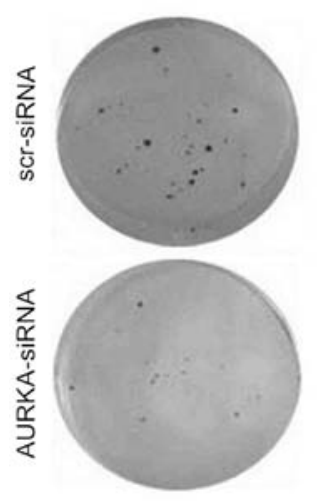

H466 cells

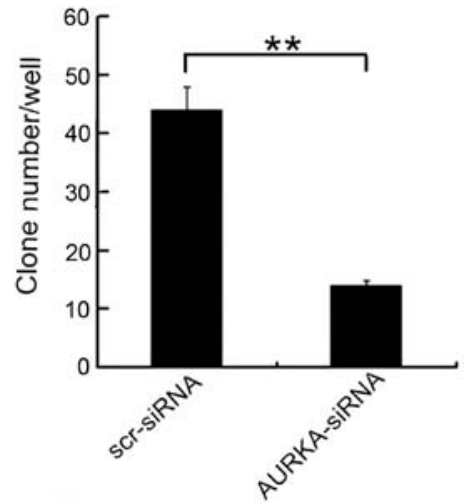

B

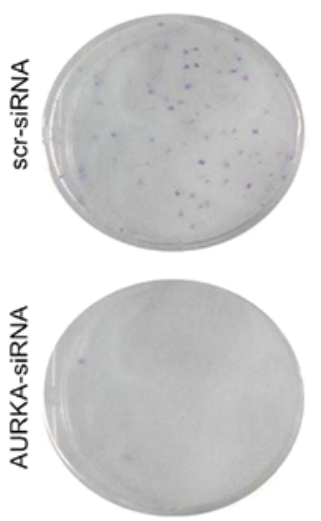

H1688 cells

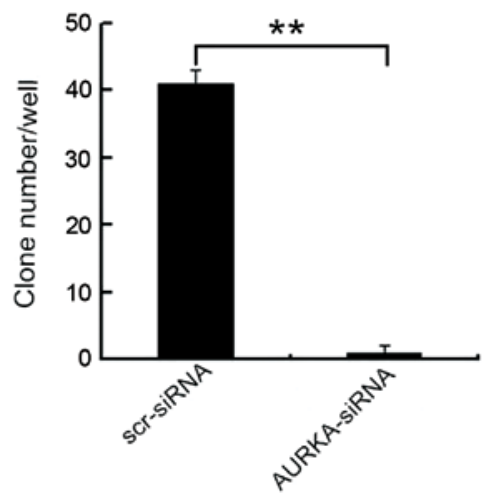

Figure 4. Inhibition of AURKA decreases colony formation of SCLC cells. Colony formation assay showed that LV-AURKA siRNA decreased the formation of colonies in H446 (A) and H1688 (B) cells. ${ }^{* *}$ p $<0.01$, compared with control treatment. AURKA, Aurora kinase A; SCLC, small cell lung cancer; siRNA, small interfering RNA.

Cell cycle profile analysis after knockdown of AURKA in $S C L C$ cells. To determine if cell growth inhibition was the upshot of a shift in the cell cycle, we examined cell cycle phase distribution of SCLC cells after the silencing of AURKA. As shown in Fig. 5, treatment with AURKA-siRNA resulted in an increase in the percentage of $\mathrm{H} 446$ cells in the G2/M phase from $3.13 \pm 0.29 \%$ to $11.62 \pm 0.19 \%$ ( $\mathrm{p}<0.01)$. Concomitant with this increase in the percentage of cells in the G2/M phase was a significant decrease in the percentage of cells in G1 phase, from $64.10 \pm 0.30 \%$ to $51.44 \pm 0.72 \%$ ( $\mathrm{p}<0.01)$. Treatment with 

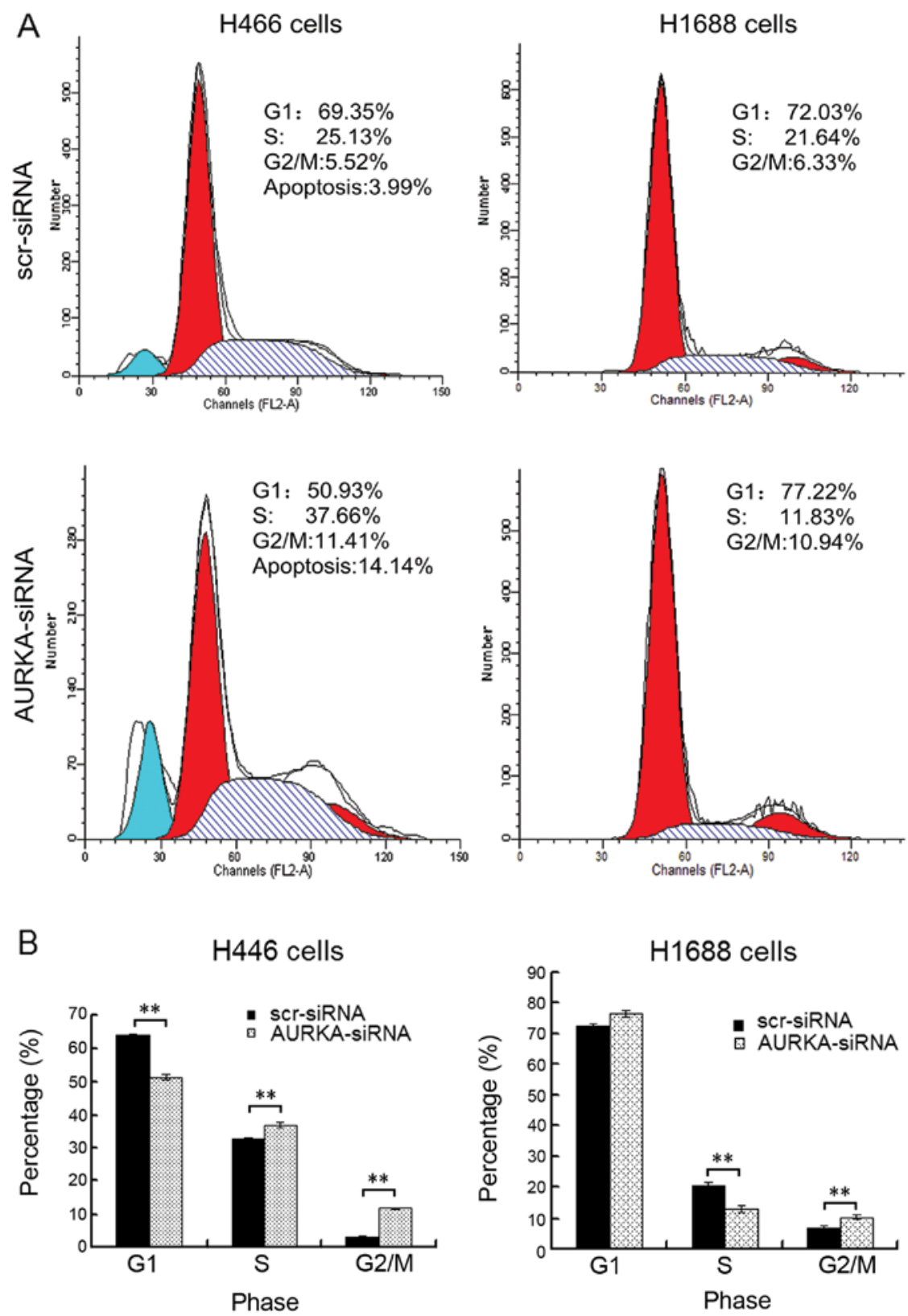

Figure 5. Effect of AURKA silencing on the cell-cycle distribution of SCLC cells. (A) The flow cytometric histogram and the number of cells under each phase of the cell cycle in both H446 and H1688 cells. (B) Numerical representation of the cells under each phase of the cell cycle in both H446 and H1688 cells. Flow cytometric analysis showed that knockdown of AURKA expression by LV-AURKA siRNA induced G2/M phase arrest to disrupt cell cycle progression. ${ }_{*}^{*}$ p $<0.01$, compared with control treatment. AURKA, Aurora kinase A; SCLC, small cell lung cancer; siRNA, small interfering RNA.

AURKA-siRNA resulted in an increase in the percentage of $\mathrm{H} 1688$ cells in the $\mathrm{G} 2 / \mathrm{M}$ phase, from $6.77 \pm 0.70 \%$ to $10.35 \pm 0.56 \%(\mathrm{p}<0.01)$. This was accompanied by a significant reduction of the fraction of $\mathrm{S}$ phase cells from $20.65 \pm 0.93 \%$ to $12.93 \pm 1.09 \%(p<0.01)$. These results together suggest that the depletion of AURKA inhibited the proliferation of SCLC cells by prompting arrest in $\mathrm{G} 2 / \mathrm{M}$ phase of the cell cycle.

To determine whether the depletion of AURKA induces cell apoptosis, flow cytometry was used to analyze the apoptosis of H446 and H1688 cells after infection with AURKA-siRNA for $72 \mathrm{~h}$. As shown in Fig. 6, flow cytometry analysis showed that the percentage of apoptotic $\mathrm{H} 446$ cells was $14.97 \pm 0.56 \%$ in scr-siRNA group and the percentage of apoptotic cells increased to $24.29 \pm 1.07 \%$ in the AURKA-siRNA group $(\mathrm{p}<0.01)$ (Fig. 6A). The percentage of apoptotic H1688 cells was $4.41 \pm 0.39 \%$ in scr-siRNA group cells and increased to $9.56 \pm 0.38 \%$ in AURKA-siRNA group $(\mathrm{p}<0.01)$ (Fig. 6B). These data suggest that the depletion of AURKA specifically induced apoptosis of the SCLC cells.

Apoptosis of H446 cells following AURKA inhibition are associated with downregulated Bcl-2 and upregulated Bax. Western blot assay showed that AURKA inhibition resulted in decreased expression of CDK1 protein, reinforcing the likelihood of G2/M cell cycle arrest in H446 cells (Fig. 7A). In order to examine the molecular mechanism of AURKA in cell apoptosis, we analyzed two key apoptotic mediators of the Bcl-2 family, Bcl-2 and Bax. Our results revealed that the knockdown of AURKA downregulated Bcl-2 and upregulated Bax expression in $\mathrm{H} 446$ cells. This result indicates that the 
A

H446 cells

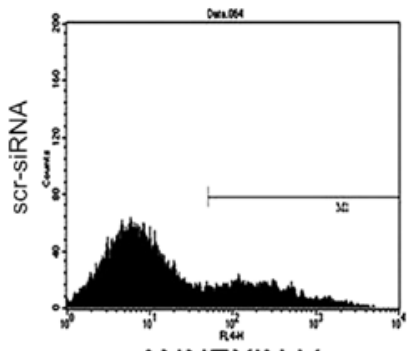

ANNEXIN-V
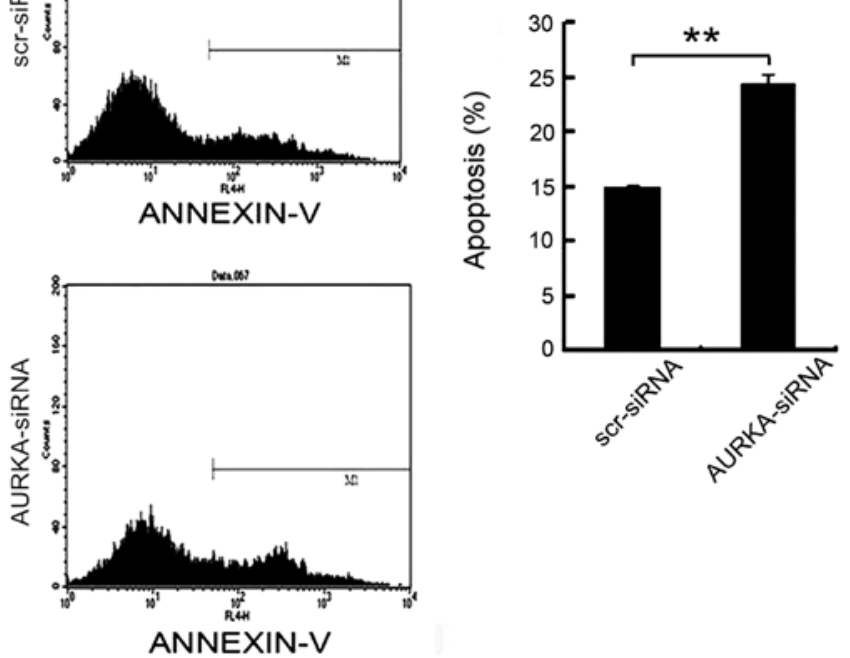

B

H1688 cells
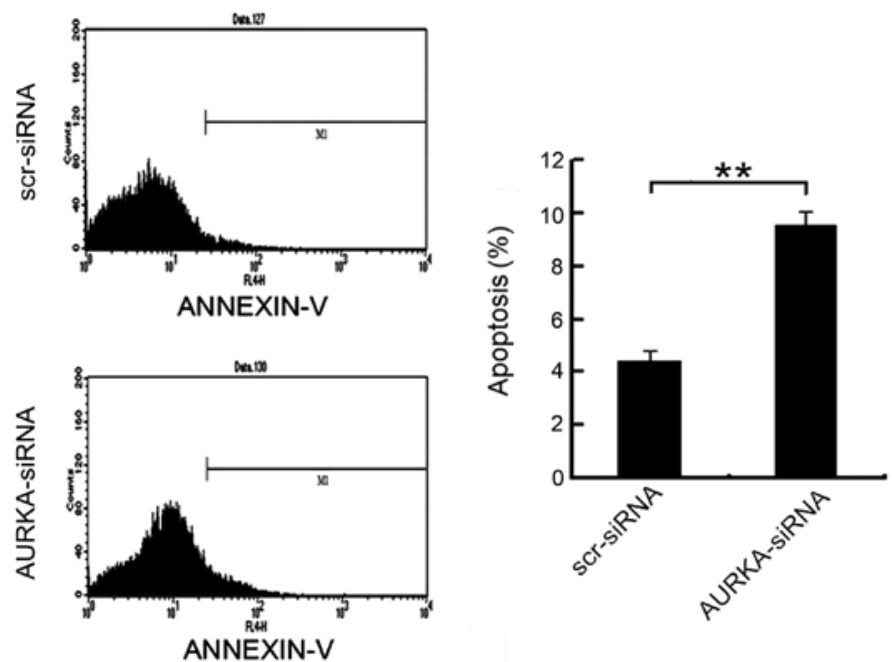

Figure 6. Effects of LV-AURKA siRNA on cell apoptosis as determined by flow cytometric analysis. Flow cytometry analysis showed that knockdown of AURKA expression via LV-AURKA siRNA increased cellular apoptosis in H446 (A) and H1688 (B) cells. ${ }^{* *}$ p $<0.01$, compared with control treatment. AURKA, Aurora kinase A; siRNA, small interfering RNA.

A

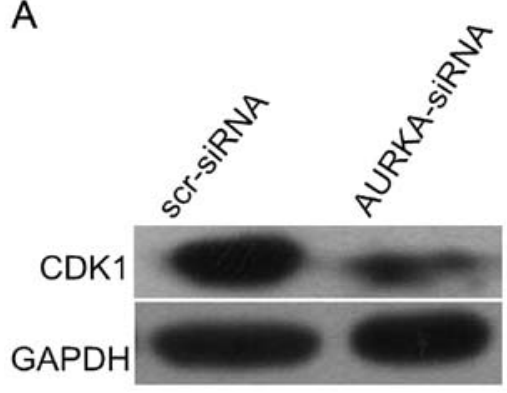

B

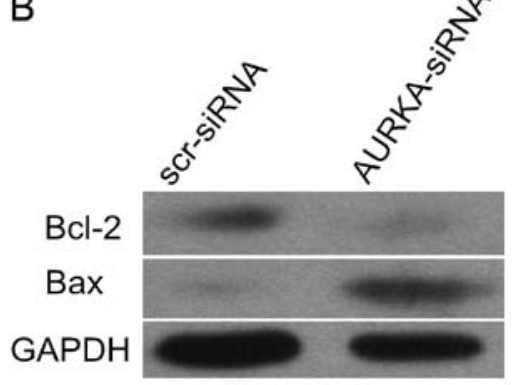

Figure 7. Western blot analysis. (A) Knockdown of AURKA expression downregulated CDK1 expression in H446-infected cells. (B) Western blot analysis of Bcl-2 family mediators of apoptosis showed that knockdown of AURKA downregulated Bcl-2 and upregulated Bax in LV-AURKA siRNA-transduced cells. AURKA, Aurora kinase A; siRNA, small interfering RNA.

heightened apoptosis associated with AURKA downregulation may be partly mediated by these $\mathrm{Bcl}-2$ family proteins in H446 cells (Fig. 7B).

\section{Discussion}

SCLC is a type of highly malignant lung tumor that metastasizes markedly quickly after its initial genesis (16). In a previous study, ectopic expression of AURKA in NIH 3T3 cells and Ratl fibroblasts led to centrosome amplification and cell transformation in vitro and manifested in tumorigenesis in vivo (17). The expression of AURKA mRNA and protein is associated with the tumor stage and metastasis in head and neck squamous cell carcinoma (18). Aneuploidy and overexpression of AURKA have been shown to predict poor outcome in serous ovarian carcinoma (19). These findings suggest that AURKA is a potential target for diagnosis and treatment for various types of cancer. However, little is known about the mechanisms of action of AURKA in SCLC.
RNA interference (RNAi) is a widely used technique to reduce the expression level of target proteins (20). The lentivirus vector is an effective vehicle for introducing RNAi into cells under controlled conditions (21). The vector is capable of integrating a vast amount of genetic information into the host's genome. The host cell permanently expresses the viral vector gene as if it was its own. We used this methodology in our study to downregulate the expression of AURKA gene, resulting in partial to complete loss of function gene (22).

In the present study, we observed that suppression of AURKA expression by AURKA-siRNA led to prompted mitotic arrest of the $\mathrm{H} 446$ and $\mathrm{H} 1688$ cells in the G2/M phase, and induced apoptosis. Other studies have found that the knockdown of AURKA inhibits tumor cell proliferation and invasion, and enhances apoptosis in other types of cancer, such as human esophageal squamous cell carcinoma (ESCC) (23). Wang et al found that the knockdown of AURKA led to increased genomic stabilization and slowed the progression of the cell cycle in addition to the aforementioned processes in ESCC cells (24). Another study, 
by Yang et al, demonstrated that the knockdown of AURKA in ovarian cancer cell lines disabled the cancer's ability to thrive, which is also through the same molecular processes (25). Our findings confirm AURKA's role in SCLC cells.

CDK1 is necessary for cells to exit the G2 phase and enter mitosis (26). CDK1 activity is determined by the relative levels of CDK1 activators and repressors in the cellular environment. The major activator of CDK1 is cyclin B1 (27). Activated AURKA is required for the recruitment of CDK1-cyclin B1 to the centrosome and thus the commitment of a cell to mitosis. Moreover, AURKA phosphorylates CDC25B at the centrosome and contributes to G2/M transition in cancer cells (28). Our findings demonstrated that AURKA inhibition resulted in decreased CDK1 protein expression. This may be associated with the noted G2/M arrest. Moreover, apoptosis is partially modulated by the Bcl-2 family, including both apoptotic enabling as well as inhibiting factors $(29,30)$. Bcl-2 and Bax are among the most widely recognized pro-survival pro-apoptotic proteins, respectively. Bcl-2 and Bax can form homodimers or heterodimers with one another (31). Reduced Bcl-2 expression in the presence of increased Bax expression likely generates a dominant signal in favor of cell death (32). In the present study, we found that the knockdown of AURKA decreased Bcl-2 expression and increased Bax expression. This indicates that the Bcl-2 family may be implicated in the apoptosis of H446 cells following knockdown of AURKA.

In conclusion, our results demonstrated that the significant downregulation of AURKA expression by AURKA siRNA in SCLC cells inhibited cell proliferation and induced cell apoptosis. A potential mechanism of the mitotic suppression was widespread arrest in the G2/M phase of the cell cycle. In addition, the increased cell apoptosis rate after knockdown of the AURKA gene may be partially through downregulation of Bcl-2 and upregulation of Bax. Our data therefore elucidate the potentially therapeutic roles of AURKA in SCLC.

\section{Acknowledgements}

This study was supported by grants from the National Natural Science Foundation of China (no. 81172347) and the Science and Technology Foundation of Kunshan city (KSZ1309). The authors thank Dr Wenxiang Wei (Soochow University, Suzhou 215123, China) for his help and technical support.

\section{References}

1. van Meerbeeck JP, Fennell DA and De Ruysscher DK: Small-cell lung cancer. Lancet 378: 1741-1755, 2011.

2. Siegel R, Naishadham D and Jemal A: Cancer statistics, 2012. CA Cancer J Clin 62: 10-29, 2012.

3. Lally BE, Urbanic JJ, Blackstock AW, Miller AA and Perry MC: Small cell lung cancer: have we made any progress over the last 25 years? Oncologist 12: 1096-1104, 2007.

4. Oze I, Hotta K, Kiura K, et al: Twenty-seven years of phase III trials for patients with extensive disease small-cell lung cancer: disappointing results. PLoS One 4: e7835, 2009.

5. Vader $\mathrm{G}$ and Lens SM: The Aurora kinase family in cell division and cancer. Biochim Biophys Acta 1786: 60-72, 2008.

6. Nikonova AS, Astsaturov I, Serebriiskii IG, Dunbrack RL Jr and Golemis EA: Aurora A kinase (AURKA) in normal and pathological cell division. Cell Mol Life Sci 70: 661-687, 2013.

7. Katayama H, Brinkley WR and Sen S: The Aurora kinases: role in cell transformation and tumorigenesis. Cancer Metastasis Rev 22: 451-464, 2003.
8. Jiang Y, Zhang Y, Lees E and Seghezzi W: AuroraA overexpression overrides the mitotic spindle checkpoint triggered by nocodazole, a microtubule destabilizer. Oncogene 22: 8293-8301, 2003.

9. Anand S, Penrhyn-Lowe S and Venkitaraman AR: AURORA-A amplification overrides the mitotic spindle assembly checkpoint, inducing resistance to Taxol. Cancer Cell 3: 51-62, 2003.

10. Zhou H, Kuang J, Zhong L, et al: Tumour amplified kinase STK15/BTAK induces centrosome amplification, aneuploidy and transformation. Nat Genet 20: 189-193, 1998.

11. Wang X, Zhou YX, Qiao W, et al: Overexpression of aurora kinase $\mathrm{A}$ in mouse mammary epithelium induces genetic instability preceding mammary tumor formation. Oncogene 25: 7148-7158, 2006

12. Burnett JC and Rossi JJ: RNA-based therapeutics: current progress and future prospects. Chem Biol 19: 60-71, 2012.

13. Watts JK and Corey DR: Silencing disease genes in the laboratory and the clinic. J Pathol 226: 365-379, 2012.

14. Castanotto D and Rossi JJ: The promises and pitfalls of RNA-interference-based therapeutics. Nature 457: 426-433, 2009.

15. Ketting RF: The many faces of RNAi. Dev Cell 20: 148-161, 2011.

16. Stovold R, Blackhall F, Meredith S, Hou J, Dive C and White A: Biomarkers for small cell lung cancer: neuroendocrine, epithelial and circulating tumour cells. Lung Cancer 76: 263-268, 2012

17. Bischoff JR, Anderson L, Zhu Y, et al: A homologue of Drosophila aurora kinase is oncogenic and amplified in human cancers. EMBO J 17: 3052-3065, 1998.

18. Reiter R, Gais P, Jütting U, et al: Aurora kinase A messenger RNA overexpression is correlated with tumor progression and shortened survival in head and neck squamous cell carcinoma. Clin Cancer Res 12: 5136-5141, 2006.

19. Lassus H, Staff S, Leminen A, Isola J and Butzow R: Aurora-A overexpression and aneuploidy predict poor outcome in serous ovarian carcinoma. Gynecol Oncol 120: 11-17, 2011.

20. Stein EV, Price DK and Figg WD: shRNA technology: investigating Ras-dependent cancer. Cancer Biol Ther 8: 1798-1799, 2009.

21. Luo J, Emanuele MJ, Li D, et al: A genome-wide RNAi screen identifies multiple synthetic lethal interactions with the Ras oncogene. Cell 137: 835-848, 2009.

22. Brummelkamp TR, Bernards R and Agami R: A system for stable expression of short interfering RNAs in mammalian cells. Science 296: 550-553, 2002.

23. Wang X, Dong L, Xie J, Tong T and Zhan Q: Stable knockdown of Aurora-A by vector-based RNA interference in human esophageal squamous cell carcinoma cell line inhibits tumor cell proliferation, invasion and enhances apoptosis. Cancer Biol Ther 8: 1852-1859, 2009.

24. Wang X,Lu N, Niu B, Chen X, Xie J and Cheng N: Overexpression of Aurora-A enhances invasion and matrix metalloproteinase-2 expression in esophageal squamous cell carcinoma cells. Mol Cancer Res 10: 588-596, 2012.

25. Yang G, Chang B, Yang F, et al: Aurora kinase A promotes ovarian tumorigenesis through dysregulation of the cell cycle and suppression of BRCA2. Clin Cancer Res 16: 3171-3181, 2010.

26. Satyanarayana A and Kaldis P: Mammalian cell-cycle regulation: several Cdks, numerous cyclins and diverse compensatory mechanisms. Oncogene 28: 2925-2939, 2009.

27. Riabowol K, Draetta G, Brizuela L, Vandre D and Beach D: The cdc2 kinase is a nuclear protein that is essential for mitosis in mammalian cells. Cell 57: 393-401, 1989.

28. Dutertre S, Cazales M, Quaranta M, et al: Phosphorylation of CDC25B by Aurora-A at the centrosome contributes to the G2-M transition. J Cell Sci 117: 2523-2531, 2004.

29. Kelly PN and Strasser A: The role of Bcl-2 and its pro-survival relatives in tumourigenesis and cancer therapy. Cell Death Differ 18: 1414-1424, 2011.

30. Qi F, Inagaki Y, Gao B, et al: Bufalin and cinobufagin induce apoptosis of human hepatocellular carcinoma cells via Fas- and mitochondria-mediated pathways. Cancer Sci 102: 951-958, 2011.

31. Oltersdorf T, Elmore SW, Shoemaker AR, et al: An inhibitor of Bcl-2 family proteins induces regression of solid tumours. Nature 435: 677-681, 2005.

32. Gu X, Yao Y, Cheng R, et al: Plasminogen K5 activates mitochondrial apoptosis pathway in endothelial cells by regulating Bak and Bcl- $\mathrm{x}_{\mathrm{L}}$ subcellular distribution. Apoptosis 16: 846-855, 2011. 